\title{
„Multitude“ und „être collectif‘: Zu einer poetologischen Denkfigur bei Goethe, Emerson und Whitman
}

\author{
Kai Sina
}

So intensiv sich die Forschung mit der Frage nach der Rezeption Walt Whitmans in der deutschsprachigen Literatur und Kultur befasst hat (weiterhin einschlägig ist hier vor allem die wegweisende Studie von Walter Grünzweig), ${ }^{1}$ so unzusammenhängend und vor allem nur oberflächlich ist bislang in umgekehrter Blickrichtung die Rezeption der deutschsprachigen Dichtung und Philosophie durch Whitman untersucht worden. Wenngleich Floyd Stovall in seiner bereits 1974 veröffentlichten Studie mit Nachdruck auf den hohen Stellenwert von Herder und Hegel, Goethe und Heine für Whitman hingewiesen hat - eine systematische und differenzierte Untersuchung dieser Einflussbeziehungen muss bis heute als Desiderat gelten. ${ }^{2}$ Um dem zumindest punktuell entgegenzuwirken, will ich den Blick auf jenen herausragenden Protagonisten des deutschen Geisteslebens richten, dessen Werk Whitman nicht nur nachweislich kannte, sondern aus dessen Ideen sich zudem

\footnotetext{
* Es handelt sich hierbei um eine gekürzte, durchgesehene und überarbeitete Fassung eines Aufsatzes, der 2013 in Comparatio. Zeitschrift für vergleichende Literaturwissenschaft erschienen ist. In meiner derzeit entstehenden Habilitationsschrift Eines aus Vielem. Genese einer kollektiven Poetik der Moderne werden die hier aufgegriffenen Thesen und Probleme noch eingehender reflektiert sowie literatur- und ideengeschichtlich kontextualisiert.

1 Walter Grünzweig: Walt Whitmann [sic!]. Die deutschsprachige Rezeption als interkulturelles Phänomen. München 1991.

2 Floyd Stovall: The Foreground of „Leaves of Grass". Charlottesville 1974, darin die Abschnitte S. 129137 (zu Goethe), S. 184-204 (zu den deutschen Philosophen) und S. 222-230 (zu Heine).
} 
grundlegende Folgen für die poetologische Konzeption der literarhistorisch außerordentlich wirkmächtigen Gedichtsammlung Leaves of Grass einschließlich des ihr eingeschriebenen Konzepts von Autorschaft ergaben - nämlich Goethe.

Die Frage nach Whitmans Auseinandersetzung mit Goethe, nach einem konkreten Einfluss auf sein Denken und seine Dichtung, ist auf den ersten Blick alles andere als naheliegend. Befragt nach seiner Haltung gegenüber Goethe, äußerte Whitman immer wieder grundlegende Skepsis, ja auch unverhohlene Ablehnung. Unmissverständlich zum Ausdruck kommt dies in einer nachgelassenen Notiz vom 18. Februar 1856:

There is one point of the Goethean philosophy which without appeal and forever incapacitates it from suiting America or the forthcoming years; - It is the cardinal Goethean doctrine too, that the artist or poet is to live in art or poetry alone apart from affairs, politics, facts, vulgar life, persons, and things - seeking his ,high ideal.“3

Nach einigen weiteren, ähnlich kritischen Anmerkungen zu Goethe und dessen Kunst- und Literaturauffassung, deren (angeblich) einsinniger Individualismus in einem unaufhebbaren Widerspruch zur geistigen Verfassung der, neuen Welt' gesehen wird, verhängt Whitman vier Tage später, am 22. Februar, sein vermeintlich letztgültiges Urteil. Aus seiner Formulierung spricht deutlich das selbstbewusste Distinktions- und Emanzipationsbestreben des jungen Amerika: „To the genius of America he [Goethe; K.S.] is neither dear nor the reverse of dear. He passes with the general crowd upon whom the American glance descends with indifference. Our road is our own." "4

Die in Gänze nicht mehr überschaubare Whitman-Forschung hat sich mit dieser Zurückweisung, soweit ich sehe, bislang zufrieden gegeben - und dies hat auf den ersten Blick durchaus seine Berechtigung. Denn in der Tat lassen sich die Vorstellungen einer ausschließlich auf die Kunst und den Künstler bezogenen Lebens-

\footnotetext{
${ }^{3}$ Walt Whitman: Notebooks and Unpublished Prose Manuscripts. Vol. 5: Notes. Hg. von Edward F. Grier. New York 1984, S. 1826.

${ }^{4}$ Ebd., S. 1828. Vgl. zu Whitmans Goethe-Bild auch eine späte Gesprächsnotiz, in der Whitman zwar das Bildungskonzept Goethes durchaus bewundernd anerkennt, um es jedoch gleich darauf wegen seiner exklusiven Ausrichtung auf das ,Ich“ zu verwerfen: „W[hitman] himself spoke of Goethe. [...] ,Goethe impresses me as above all to stand for essential literature, art, life - to argue the importance of centering life in self - in perfect persons - perfect you, me: to force the real into the abstract ideal: to make himself, Goethe, the supremest example of personal identity: everything making for it: in us, in Goethe: every man repeating the same experience.' Goethe would ask: ,What are your forty, fifty, hundred, social, national, phantasms? This only is real - this person.' [...] ,Goethe seemed to look upon personal development as an end in itself: the old teachers looked for collective results. I do not mean that Goethe was immoral, bad - only that he laid stress upon another point. Goethe was for beauty, erudition, knowledge - first of all for culture. I doubt if another imaginist of the first order in all literature, all history, so deeply put his stamp there. Goethe asked, What do you make out of your patriotism, army, state, people?' It was all nothing to him“" (Horace Traubel: With Walt Wbitman in Camden. Vol. 3: November 1, 1888 - January 20, 1889. New York 1914, S. 159f.).
} 
form, die Goethe hier in entstellender Vereinfachung zugeschrieben wird, mit Whitmans Poetik zunächst kaum vereinbaren; für den, wahren' Künstler gelte schließlich vielmehr, wie Whitman im Jahr 1888 in einem Vorwort zu einer erneuten Auflage der Leaves betont, ,what Herder taught to the young Goethe, that really great poetry is always (like the Homeric or Biblical canticles) the result of a national spirit, and not the privilege of a polish'd and select few ". ${ }^{5}$ Folgt man darüber hinaus der bisweilen grellen Stilisierung Whitmans zum „,nationale[n] Kosmos“, 6 dessen herausgehobene Stellung sich ausschließlich im Kontext der amerikanischen Kultur in ihrem Streben nach Unabhängigkeit von europäischen Vorbildern entfaltet, so liegt es zunächst denkbar fern, ausgerechnet im fernen ,Ilm-Athen ${ }^{6}$ nach möglichen Einflüssen, vielleicht gar Wurzeln seiner Poetik zu suchen.

Nun ist Whitmans Bemerkung, er habe zwar eine Meinung zu Goethe, wisse aber so gut wie nichts über ihn, ${ }^{7}$ im Lichte der Forschung entschieden zurückzuweisen. Schon Stovall stellt hierzu fest, Whitmans Interesse an Goethe habe zwischen 1846 und seinem Tod im Jahr 1892 eigentlich zu keinem Zeitpunkt wirklich nachgelassen. ${ }^{8}$ Der Beginn dieser fast vierzigjährigen Beschäftigung mit Goethe lässt sich für Stovall dergestalt klar datieren, weil Whitman in diesem und im darauf folgenden Jahr die von Parke Godwin übersetzte Autobiography of Goethe. Truth and Poetry: From my Life (Band 1 und 2 erschienen 1846, Band 3 und 4 erschienen 1847) las und ihr sogar zwei kurze und begeisterte Besprechungen im Brooklyn Daily Eagle widmete (,the simple easy truthful narrative of the existence and experience of a man of genius"). ${ }^{9}$ Außerdem befasste sich Whitman nachweislich - wahrscheinlich im Jahr 1857, möglicherweise aber auch schon etwas früher - mit Thomas Carlyles Critical and Miscellaneous Essays (erstmals publiziert 1838-39, dann erneut in einem Einzelband im Jahr 1845), von denen sich allein sechs Stücke mit Goethe befassen. ${ }^{10}$ Und nicht zuletzt bestätigen zahlreiche Notizen auf unterschiedlichen Papieren im handschriftlichen Nachlass Whitmans fortwährendes Interesse an Goethe. ${ }^{11}$

Aber so langanhaltend und eingehend sich die Auseinandersetzung Whitmans mit Goethe aus philologischer Perspektive darstellt - in letzter Konsequenz lief sie scheinbar bloß auf jene eingangs umrissene harsche und zudem oberflächliche

\footnotetext{
5 Walt Whitman: „Leaves of Grass“ and Other Writings. Hg. von Michael Moon. New York/London 2002, S. 484.

${ }^{6}$ So das seither vielfach fortgeschriebene Label dieses Autors; vgl. beispielhaft Rolf Geisler: [Art.] Whitman, Leaves of Grass. In: Kindlers Neues Literaturlexikon. Hg. von Walter Jens. München 1998, Bd. 17, S. $611 \mathrm{ff} .$, hier S. 612.

7 Das entsprechende Zitat findet sich im oben angegebenen Gespräch mit Traubel: „W[hitman] stopped and laughed. ,So you see I have an opinion while I confess I know nothing about Goethe."“ (Traubel: With Walt Whitman in Camden, S. 160).

8 Vgl. Stovall: The Foreground of „Leaves of Grass“, S. 129-137, hier S. 132.

9 Beide abgedruckt in The Uncollected Poetry and Prose of Walt Whitman. Hg. von Emory Holloway. Garden City, N.Y./Toronto 1921, Bd. 1, S. 132 und S. 139ff., hier S. 140. Die Besprechung des ersten Bandes nimmt in Holloways Edition - mit umfangreicher Erläuterung der Herausgeberin in der Fußnote - nur knapp eine Druckseite in Anspruch, während die Besprechung des zweiten Bandes nur sieben Druckzeilen umfasst.

${ }^{10}$ Stovall: The Foreground of „Leaves of Grass", S. 132.

${ }^{11}$ Ebd., S. $133 \mathrm{f}$.
} 
Abgrenzung hinaus, die auf längere Sicht dazu führte, dass mögliche Beziehungen im Denken und Schreiben von Whitman und Goethe kaum in den Fokus der Forschung geraten sind. Anmerkungen wie die von Stovall, wonach Goethes Autobiografie nicht nur „the conception“, sondern möglicherweise auch „the composition" der Leaves bestimmt habe, ${ }^{12}$ sind eher die Ausnahme.

Dabei hätte schon die betonte Ausdrücklichkeit in Whitmans Abgrenzung aufhorchen lassen können, vielleicht müssen: Warum betont Whitman überhaupt eine so klare Distanz, wenn Goethe doch in einer allgemeinen Masse von Dichtern untergehe, „upon whom the American glance descends with indifference"? Die bloße Artikulation dieses Satzes verfängt sich in einem Selbstwiderspruch. Und damit der Auffälligkeit nicht genug. Richtet man den Blick außerdem auf Whitmans Poetik im engeren Sinne, so lässt sich eine literaturgeschichtlich bedeutsame, von der bisherigen Forschung allerdings nicht angemessen gewürdigte, ja nicht einmal ausdrücklich benannte Überschneidung mit dem dichterischen Selbstverständnis Goethes benennen: Es handelt sich um die Idee vom Autor als einem Medium, in dessen einer Stimme sich die Stimmen vieler Menschen zum Ausdruck bringen. „, [M]ein Lebenswerk ist das eines Kollektivwesens, und dies Werk trägt den Namen Goethe", ${ }^{13}$ so bekennt der Weimarer Dichter kurz vor seinem Tod in einem persönlichen Gespräch. Etwas mehr als zwei Jahrzehnte später wird sich Whitman in dem berühmten Song of Myself folgendermaßen charakterisieren: „I am large .... I contain multitudes. "“14 Hierbei handelt es sich keineswegs - so meine These - um eine nur akzidentelle, punktuelle Kongruenz zweier poetologischer Konzepte. Vielmehr liegt es nahe, von einem konkreten Einfluss Goethes auf Whitman auszugehen, der sich allerdings nicht über eine direkte Lektüre vollzog, sondern über eine vermittelte Rezeption.

Die Essays von Ralph Waldo Emerson las Whitman erstmals im Sommer $1854,{ }^{15}$ und er stellte im Rückblick über diese Lektüre fest, erst durch sie habe er zu sich gefunden: „I was simmering, simmering, simmering; Emerson brought me to a boil.““16 Als mögliche Impulse für die parallel zu diesen Lektüren entstehenden

\footnotetext{
12 Ebd., S. 129.

13 Johann Wolfgang Goethe: Die letsten Jahre. Briefe, Tagebücher und Gespräche von 1823 bis zu Goethes Tod. Teil III: Vom Dornburger Aufenthalt 1828 bis zum Tode. Hg. von Horst Fleig. Frankfurt a.M. 1993, S. $521 \mathrm{f}$.

${ }^{14}$ Hier zitiert nach der Erstausgabe der Leaves von 1855 (Whitman: Leaves of Grass, S. 662-751). Künftig werde ich die Quellen- bzw. Versangaben direkt im Fließtext angeben (hier V. 1316).

${ }^{15}$ Den entscheidenden - und von der Forschung grundsätzlich als glaubwürdig erachteten - Hinweis hierauf formulierte John Townsend Trowbridge in seinem Text Reminiscences of Walt Whitman. Der ursprünglich im Februar 1902 in der Zeitschrift The Atlantic Monthly publizierte Text ist im Internet abrufbar: <http://www.theatlantic.com/past/docs/unbound/poetry/whitman/walt.htm> Die Konstellation Emerson/Whitman ist so eingehend und ausführlich erforscht, dass hier der Verweis auf zwei neuere Publikationen hinreichen möge; einerseits auf John Michael Corrigan: American $\mathrm{Me}$ tempsychosis. Emerson, Whitman, and the New Poetry. New York 2012, andererseits auf Jay Grossmann: Reconstituting the American Renaissance. Emerson, Whitman, and the Politics of Representation. Durham/London 2003.

16 Dieses pointierte Zitat entstammt freilich der Erinnerung von Trowbridge: Reminiscences of $W$ alt Whitman.
} 
und ein Jahr später in ihrer Erstfassung publizierten Leaves werden in der Forschung unterschiedliche Essays Emersons diskutiert, allen voran The Poet. ${ }^{17}$ Aber auch Emersons Essaysammlung Representative Men, die eine Reihe von Autorenporträts in Buchform versammelt, wird als eine mögliche Inspirationsquelle genannt, ${ }^{18}$ und das letzte Kapitel in diesem Band ist nun wiederum jenem Dichter gewidmet, der das Denken Emersons seinerseits tief und anhaltend geprägt hat, nämlich Goethe. ${ }^{19}$ In seinem Essay Goethe; or, the Writer vollzieht Emerson eine Umdeutung des von Whitman als individualitätsfixiert verworfenen Kunst- und Persönlichkeitskonzepts Goethes, indem er dem Dichter die gesellschaftlich unerlässliche Funktion als Allversöhner einer dissoziierten Moderne zuschreibt. In dieser romantisierenden Lesart, so wird im Folgenden zu zeigen sein, wirkt Goethes Idee auf Whitmans Poetik auf ebenso vermittelte wie grundlegende Weise ein.

Aber es geht es mir nicht allein um den Nachweis, dass ein wichtiger Aspekt in Whitmans Poetik auf einen Einfluss Goethes zurückzuführen ist. Über diese bloß werkgenetische und daher recht eng eingestellte Optik der Einflussforschung hinaus will ich eine Interpretation versuchen, die auf folgende zwei Fragen Antworten zu geben sucht: 1.) Wenn sich tatsächlich ein über Emerson vermittelter Einfluss Goethes auf Whitmans Poetik nachweisen lässt - warum negiert er diese Bezogenheit in seinen poetologischen Selbstaussagen so vehement? Und was sagt dies 2.) über das Konzept seiner vermeintlich autochthonen Nationalpoesie aus, die gemeinhin als Grundstein einer originär amerikanischen modernen Dichtung angesehen wird? Die Durchführung der Untersuchung folgt dabei naheliegender Weise der zu rekonstruierenden Einflussbeziehung - von Goethe ausgehend dem Umweg über Emerson folgend schließlich zu Whitman.

\section{Goethe}

In einem längeren Gespräch mit dem Schweizer Numismatiker und Privatgelehrten Frédéric Soret, das Goethe am 17. Februar 1832, also nur knapp einen Monat vor seinem Tod, im Haus am Frauenplan führte, bekannte sich dieser zu einer kollektiven Kontur seines Lebenswerks:

\footnotetext{
${ }^{17}$ Vgl. für einen Nachweis zahlreicher Parallelstellen Stovall: The Foreground of „Leaves of Grass“, S. 296304.

${ }^{18}$ Zur besonderen Bedeutung der Representative Men für Whitman - wenn auch nicht zu dem darin enthaltenen Goethe-Essay - Joel Porte: Representative Man. Ralph Waldo Emerson in His Time. New York 1979, S. 314ff.

${ }^{19}$ Die Bedeutung Goethes für Emerson, die Kon- und Divergenzen ihres Schreibens und Denkens sind eingehend und umfassend erforscht. Vgl. an neueren Publikationen hier nur Peter A. Obuchowski: Emerson and Science. Goethe, Monism, and the Search for Unity. Great Barrington 2005 und Philipp Mehne: Bildung vs. Self-Reliance? Selbstkultur bei Goethe und Emerson. Würzburg 2008. Eine genuin literarische und daher gesondert erwähnenswerte Verbindung von Emerson zu Goethe beschreibt J. Lasley Dameron: Emerson's „Each and All" and Goethes „Eins und Alles". In: English Studies. A Journal of English Language and Literature 67, 4 (1986), S. 327-330.
} 
Was habe ich denn gemacht? Ich sammelte und benutzte alles was mir vor Augen, vor Ohren, vor die Sinne kam. Zu meinen Werken haben Tausende von Einzelwesen das ihrige beigetragen, Toren und Weise, geistreiche Leute und Dummköpfe, Kinder, Männer und Greise, sie alle kamen und brachten ihre Gedanken, ihr Können, ihre Erfahrungen, ihr Leben und ihr Sein; so erntete ich oft, was andere gesät; mein Lebenswerk ist das eines Kollektivwesens, und dies Werk trägt den Namen Goethe. ${ }^{20}$

Entscheidend ist zunächst, worauf sich Goethe mit diesen Sätzen gerade nicht bezieht, nämlich auf das Phänomen kollektiver Autorschaft, das er ebenfalls an unterschiedlichen Stellen anspricht, vor allem mit Bezug auf seine Tätigkeit als Rezensent und seine Lyriksammlungen - und die er selbst durchaus intensiv praktizierte, was in der Forschung bereits mehrfach und eingehend dargelegt worden ist. ${ }^{21}$ Goethes Worte über das „Kollektivwesen“ richten den Fokus hingegen auf andere Aspekte. Die hier zitierte Aussage enthält - in einer aperçuhaften Verdichtung, wie sie für das Spätwerk Goethes insgesamt charakteristisch ist ${ }^{22}-$ mindestens sechs grundsätzliche Aussagen über den Schriftsteller, die poetische Praxis und das literarische Werk:

Erstens entwirft Goethe den Dichter als einen Menschen, der nicht allein aus sich selbst heraus schöpft, sondern die herausragende Fähigkeit besitzt, sämtliche Eindrücke, die ihm „,vor Augen, vor Ohren“ kommen, produktiv weiter zu verwenden, und das bedeutet hier: in Kunst zu verwandeln. Diese poetologische Denkfigur impliziert zweitens ein Modell literarischer Produktivität: Das „Genie“ nimmt seine gesamte Lebenswirklichkeit zunächst in sich auf, um sie daraufhin, als eine Art Filter, in sein Werk eingehen zu lassen, und zwar in umgewandelter, gleichsam zur vollen Reife gebrachter Form („so erntete ich oft, was andere ge$\left.s a ̈ t^{\circ}\right)$. Kennzeichnend für diesen Ansatz ist drittens eine ebenso egalitäre wie universelle Ausrichtung: Schlichtweg „alles“, was dem kunstschaffenden Genie begegnet - sei es persönlicher, gegenständlicher oder geistiger Natur, sei es geistreich oder alltäglich, sei es alt oder jung - geht über das „Kollektivwesen“ in das Werk ein. Dieser Gedanke wiederum legt viertens nahe, dass der Autor mit einer gewissen (an dieser Stelle allerdings nicht konkret benannten) repräsentativen Geltung ausgestattet ist: Er ist es, der für Viele und für Vieles spricht, die und das Teil seines Selbst und damit seines Kunstwerks geworden sind. Auf den damit verbundenen Aspekt der Verkörperlichung kommt es fünftens an: Das „Können“, die „Erfahrungen“, das

\footnotetext{
${ }^{20}$ Goethe: Die letzten Jahre, S. $521 \mathrm{f}$.

$21 \mathrm{Vgl}$. hierzu zuletzt und mit weiteren Nachweisen die Studie von Franziska Lenz: Kollektive Arbeitsweisen in der Lyrikproduktion von Goethe: „Nur durch Aneignung fremder Schätze entstebt ein Großes “. Würzburg 2013.

22 Vgl. zur Formenvielfalt des Aperçu in Goethes Spätwerk die anregenden Überlegungen von Hermann Schmitz: Goethes Altersdenken im problemgeschichtlichen Zusammenhang. Bonn 2008 (Reprint der Erstausgabe von 1959), S. 168-179. Schmitz geht auch auf die verschiedenen Selbstaussagen Goethes zur Form des Aperçu ein.
} 
„Leben“ und das „Sein“, das „Tausende von Einzelwesen“ mit sich bringen, all diese einzelnen Elemente werden Teile eines organisch gedachten Gesamtzusammenhangs („Kollektivwesen“), der sich darüber hinaus in einer namentlich bestimmbaren Person („Goethe") konkretisiert - womit schließlich und sechstens ein Modell von prozessualer und infinit gedachter Selbstkultivierung, kurz: von ,Bildung' impliziert wird.

Diese sowohl ästhetisch-poetologischen als auch ethisch-anthropologischen Überlegungen sind für Goethes Spätwerk von zentraler Bedeutung - und in der Vergangenheit bereits so eingehend und umfangreich untersucht worden, dass im Folgenden einige kursorische Bemerkungen zur werkgeschichtlichen Kontextualisierung hinreichen mögen. Zusammenfassen lassen sich unter der Zentralformel des „Kollektivwesens“ zunächst sowohl Goethes naturwissenschaftliche Bemühungen - vor allem in der Farbenlehre mit ihrer Integration unterschiedlicher, sowohl mathematischer als auch metaphysischer, mechanischer und moralischer Vorstellungsarten - wie auch sein narrativer und lyrischer Altersstil, wie er in den Wablverwandtschaften, dem zweiten Teil des Faust und in Wilhelm Meisters Wanderjahren, in der Trilogie der Leidenschaft und den ,weltliterarischen' Gedichten des Westöstlichen Divan oder der Chinesisch-deutschen Jahres- und Tageszeiten Gestalt gefunden hat. Die Forschung erkennt zwischen den beiden Werkebenen - Goethes eigener Setzung folgend - ein unmittelbares Entsprechungsverhältnis, wodurch nicht zuletzt eine allgemeine epochengeschichtliche Einordnung nahegelegt wird:

Was im Feld der naturwissenschaftlichen Naturerkenntnis durch den Ansatz einer differenzbewußten Interdisziplinarität zum Ausdruck kommt, findet [...] seine Entsprechung in einem polyperspektivischen Darstellungsverfahren, das unterschiedliche Sichtweisen aufnimmt und kontrastierend gegenüberstellt. Auch im Bereich der Fiktion setzt sich Goethe über den Zerfall des modernen Weltbildes in partikulare Perspektiven nicht hinweg, er gestaltet ihn vielmehr phantasmatisch aus, um seine Konsequenzen sichtbar zu machen. ${ }^{23}$

Für Emerson ist genau dieser Ansatz - die avancierte Ausgestaltung der Moderne in ihrer unübersehbaren Partikularität - von größter Faszinationskraft.

\section{Emerson}

Nicht allein an Goethes literarischen und autobiografischen Werken, seinen naturwissenschaftlichen und -philosophischen Arbeiten zeigte Emerson nachdrückliches und anhaltendes Interesse, sondern auch, ja vielleicht sogar mehr noch an der

${ }^{23}$ Peter Matussek: Goethe. Zur Einführung. 2., verbesserte Auflage Hamburg 2002, S. 169. 
Funktionsweise seines Geistes: „He was discernibly fascinated with the way Goethe's mind worked“, stellt Robert D. Richardson in diesem Sinne fest. ${ }^{24}$ Dass Goethes spätes Selbstbekenntnis als „Kollektivwesen“ in ganz besonderem Maße die Aufmerksamkeit des Essayisten auf sich zog, liegt aus dieser Sicht also nahe. Aber mehr noch: Das übersetzte Zitat, das Emerson im dritten Band der von Sarah Austin besorgten Characteristics of Goethe gefunden hat, ${ }^{25}$ wird für ihn zu einer zentralen, wiederholt und mit großer Zustimmung angeführten Referenzstelle. So findet es sich nicht nur in einer ausführlichen Lektürenotiz, die auf das Jahr 1834 datiert, ${ }^{26}$ und in einem Vortrag über Geoffrey Chaucer von 1835, ${ }^{27}$ sondern auch in einem 1876 veröffentlichten Essay mit dem einschlägigen Titel Quotation and Originality. ${ }^{28}$ „Goethe frankly said“, so leitet Emerson das Zitat dort ein, um daraufhin das Wort zu übergeben:

What would remain to me if this art of appropriation were derogatory to genius? Every one of my writings has been furnished to me by a thousand of different persons, a thousand things: wise and foolish have brought me, without suspecting it, the offering of their thoughts, faculties and experience. My work is an aggregation of beings taken from the whole of Nature; it bears the name of Goethe. ${ }^{29}$

Mit Goethe formuliert Emerson also die aus heutiger Sicht fast schon poststrukturalistisch anmutende Idee, dass ein Gedanke immer schon das Zitat eines anderen, früheren Gedankens , aus unterschiedlichsten Stätten der Kultur“ darstellt. ${ }^{30}$ Blickt man von hier aus auf die Representative Men und dabei insbesondere auf das Stück Goethe; or, the Writer, so wird deutlich, dass Emerson keineswegs von einem Zitat im buchstäblichen Sinne ausgeht, wenn er von „Quotation“ spricht, sondern - ganz im Sinne Goethes - von einer Umwandlung des Vorgefundenen, oder genauer:

\footnotetext{
${ }^{24}$ Vgl. Robert D. Richardson Jr.: Emerson. The Mind on Fire. Berkeley, Los Angeles/London 1995, S. 222 .

${ }^{25}$ Sarah Austin: Characteristics of Goethe: From the German of Falk, von Müller, etc. London 1833, Bd. 3, S. 74-77.

${ }^{26}$ Ralph Waldo Emerson: Journals and Miscellaneous Notebooks. Vol. VI: 1824-1838. Hg. von Ralph H. Orth. Cambridge, Mass. 1966, S. 113. Hier findet sich das Zitat in ganzer Länge. Ein späterer Eintrag greift den Kernsatz von Goethe noch einmal auf: ,What is genius but the faculty of seizing \& turning to account every thing that strikes us; of coordinating \& breathing life into all the materials that present themselves?" (ebd, S. 195).

${ }^{27}$ Ralph Waldo Emerson: Chaucer, in: The Early Lectures of Ralph Waldo Emerson. Vol. I: 1833-1836. Hg. von Stephen E. Whicher und Robert E. Spiller. Cambridge, Mass. 1966, S. 269-286.

${ }^{28}$ Das Zitat findet sich noch nicht in der 1868 erstmals in der North American Review veröffentlichten Version, wohl aber in der Erstausgabe von Letters And Social Aims (1876), der auch die hier verwendete Textausgabe folgt (Ralph Waldo Emerson: Quotation and Originality. In: Emerson's Prose and Poetry. Ausgewählt und hg. von Joel Porte und Saundra Morris. New York/London 2001, S. 319-330.

${ }^{29}$ Emerson: Quotation and Originality, S. 329.

${ }^{30}$ Roland Barthes: Der Tod des Autors. In: Texte zur Theorie der Autorschaft. Hg. von Fotis Jannidis, Gerhard Lauer [u.a.]. Stuttgart 2000, S. 185-193, hier S. 190f.
} 
von einer Erneuerung und Verfeinerung, die hier zugleich einer Art Verlebendigung entspricht:

$[\Pi \mathrm{n}$ man, the report is something more than print of the seal. It is a new and finer form of the original. The record is alive, as that which it recorded is alive. In man, the memory is a kind of looking-glass, which, having received the images of surrounding objects, is touched with life, and disposes them in a new order. [...] The man cooperates. ${ }^{31}$

Ausgehend von dieser Grundannahme einer Erneuerung, Verfeinerung („,new and finer form") und Verlebendigung (,The record is alive") des in der Erinnerung Gespeicherten, wendet sich Emerson sodann dem Dichter zu, dem er „exalted powers for this second creation“ 32 zuspricht. Er hebt dabei mit besonderem Nachdruck jenen egalitären und universellen Zug hervor, der auch Goethes Rede vom „Kollektivwesen“ eignet:

Whatever he beholds or experiences, comes to him as a model, and sits for its picture. [...] He believes that all that can be thought can be written, first or last; [...]. Nothing so broad, so subtle, or so dear, but comes therefore commended to his pen, - and he will write. In his eyes, a man is the faculty of reporting, and the universe is the possibility of being reported. In conversation, in calamity, he finds new materials $[\ldots] .{ }^{33}$

Nun kann es kaum überraschen, dass Emerson ein herausragendes Beispiel für dieses Autorschaftskonzept bei gerade jenem Dichter findet, an dessen Aussage es offenbar von vornherein geschult war. Zugleich aber geht er über Goethes Argumentation entschieden hinaus, wenn er dessen Idee vom „Kollektivwesen“ in einem romantischen Sinne funktionalisiert: ${ }^{34}$ Goethe vollziehe in seiner künstlerischen Erneuerung, Verfeinerung, Verlebendigung des mannigfach Vorgefundenen nichts Geringeres, so Emerson, als die Re-Synthetisierung einer Moderne, die in einen unübersichtlichen, allumfassenden und sich beständig ausdehnenden Pluralismus zerspalten ist, in Mythologien, Philosophien, Literaturen - und so fort.

\footnotetext{
${ }^{31}$ Ralph Waldo Emerson: Goethe; or, the Writer. In R.W.E.: Essays \& Lectures. Hg. von Joel Porte. New York 1983, S. 746-761, hier S. 746.

32 Ebd., S. 746.

${ }^{3}$ Ebd., S. 747.

${ }^{34}$ Vgl. zur eingehend und umfangreich erforschten Anverwandlung der europäischen Romantik bei Emerson, als Hauptvertreter des so genannten American Romanticism, die Studie von David Greenham: Emerson's Transatlantic Romanticism. Houndmills/Basingstoke [u.a.] 2012 (mit weiteren Literaturhinweisen).
} 
Der Dichter erscheint somit gewissermaßen als die personifizierte „Over-Soul“, als „the eternal ONE“, in dem sich die „part" und „particle“ der modernen Lebenswelt auf wunderbare Weise zur neuen Einheit fügen: ${ }^{35}$

The world extends itself like American trade. We conceive Greek or Roman life, - life in the Middle Ages, - to be a simple and comprehensible affair; but modern life to respect a multitude of things which is distracting. Goethe was the philosopher of this multiplicity; hundred-handed, Argus-eyed, able and happy to cope with this rolling miscellany of facts and sciences, and, by his own versatility, to dispose of them with ease [...]. The Helena, or the second part of Faust, is a philosophy of literature set in poetry; the work of one who found himself the master of histories, mythologies, philosophies, sciences, and national literatures [...]; and every one of these kingdoms assuming a certain aerial and poetic character, by reason of the multitude. [...] He was the soul of his century. [...] He had a power to unite the detached atoms again by their own law. He has clothed our modern existence with poetry. ${ }^{36}$

Auffällig erscheint die Wechselseitigkeit, mit der hier von der Vielheit im modernen Sinne die Rede ist: einmal als ein Negativzustand (,a multitude of things which is distracting"), der zumindest in der Kunst aufgehoben und kompensiert werden könne und solle; dann aber auch als eine Produktivkraft des Dichters, dessen Genie sich erst durch die Aufnahme und Verarbeitung dieser Vielheit entfalte, was ihn zugleich (,soul of his century") der Klasse der Representative Men zuordnet, die Emerson in seinem Band in insgesamt sechs Essays porträtiert. ${ }^{37}$

An dieser Stelle schränkt Emerson zugleich aber ein: Die vereinigende, versöhnende Aufgabe, die dem repräsentativen Dichter in seiner doppelten Stellung gegenüber der ,multitude` zugewiesen wird, sei gegenwärtig niemand zu übernehmen bereit oder auch nur imstande. Fest in die Gesellschaft integriert, komme den Dichtern das Bewusstsein ihrer ,heiligen' Aufgabe für die Gesellschaft, die Emerson im Modus spätromantischer Kunstreligion umreißt, ${ }^{38}$ nicht einmal in den Sinn:

Society has really no graver interest than the well-being of the literary class. [...] There have been times when he was a sacred person: he wrote Bibles; the first hymns; the codes; the epics, tragic songs, Sibylline verses, Chaldean oracles, Laconian sentences, inscribed on temple walls. Every word was

\footnotetext{
${ }^{35}$ Emerson: Prose and Poetry, S. 164 (The Over-Soul).

${ }^{36}$ Emerson: Goethe; or, the Writer, S. $751 \mathrm{f}$.

${ }^{37}$ Neben Goethe werden hier Platon, Swedenborg, Montaigne, Shakespeare und Napoleon behandelt.

${ }^{38}$ Zum Gegenstand der Kunstreligion siehe eingehend die Begriffsbestimmung von Heinrich Detering: Was ist Kunstreligion? Systematische und historische Bemerkungen. In: Kunstreligion. Der Ursprung des Konzepts um 1800. Hg. von Albert Meier, Alessandro Costazza [u.a.]. Berlin/New York 2011, S. 11-27.
} 
true, and woke the nations to new life. He wrote without levity, and without choice. [...] But how can he be honored, when he does not honor himself, when he loses himself in a crowd [...], ducking to the giddy opinion of a reckless public [...]; or write conventional criticism, or profligate novels; or, at any rate, write without thought, and without recurrence by day and by night, to the sources of inspiration? ${ }^{39}$

Mit seiner Klage über die Verweltlichung der Autorschaft bereitet Emerson den Boden für den Auftritt des selbsterklärten Nationaldichters Walt Whitman, der sich eben jene kunstreligiöse Emphase, die Emerson den heiligen alten, gegenwärtig aber schmerzlich vermissten Dichtern zuspricht, denn auch ganz unbescheiden selbst zu eigen macht. ${ }^{40}$ Im poetologischen Kernstück der Leaves of Grass, dem Song of Myself, kommt dies unmissverständlich zum Ausdruck; wir hören ein SprecherIch, das sich selbst als „Walt Whitman“ (V. 496) bezeichnet und sich den Lesern mit folgenden Versen vorstellt: „Divine am I inside and out, and I make holy whatever I touch or am touched from“ (V. 523).

Nun könnte dieses poetologische Komplementärverhältnis für sich genommen natürlich dem Zufall geschuldet sein (und zudem aus Emersons Essay The Poet mit seiner ins Religiöse reichenden Hypostasierung des Dichters herrühren), ${ }^{41}$ stünde sie nicht im Zusammenhang mit weiteren, signifikanten Konvergenzen, die sich auf Emerson - und von ihm ausgehend auf Goethe - zurückführen lassen.

\section{Whitman}

In Whitmans Gedicht findet sich die Betonung einer nicht-originellen, sondern auf sinnlicher Wahrnehmung der gesamten Lebensumwelt beruhenden Poesie. „O I perceive after all so many uttering tongues! / And I perceive they do not come from the roofs of mouths for nothing“ (V. 110-111), so merkt der Sprecher an, um etwas später ausdrücklich festzuhalten, die Gedanken, die er in sich aufnehme und in seinem Gesang entfalte, ,the thoughts of all men in all ages and lands, they are not original with me“ (V. 353). Das heißt, die Gedanken des Sprechers sind, mit Emerson gesprochen, nur eines, nämlich „Quotation“. Das sensorische Erfassen und, mehr noch, die sich gleichsam vegetativ vollziehende Wahrnehmung der menschlichen, geistigen und materialen Welt in ihrem gesamten Umfang umschreibt Whitman in diesem Zusammenhang zweimal mit dem Prädikat „breathe“ - „breathe the fragrance“ (V. 7), ,breathe the air“ (V. 348). ${ }^{42}$ Es ist nahezu dieselbe

\footnotetext{
${ }^{39}$ Emerson: Goethe; or, the Writer, S. 750.

${ }^{40} \mathrm{Vgl}$. eingehend zu Whitman im Kontext prophetischer Rede Bernadette Malinowski: „Das Heilige sei mein Wort". Paradigmen prophetischer Dichtung von Klopstock bis Whitman. Würzburg 2002, S. 363-406.

${ }^{41} \mathrm{Vgl}$. die Parallelstellen-Lektüre von Stovall: The Foreground of „Leaves of Grass", S. $296 \mathrm{f}$.

${ }^{42} \mathrm{Vgl}$. zu diesem als Verständnis des „,self“ als ,, a sort of lung, inhaling and exhaling the world“ Lewis Hyde: The Gift - Imagination and the Erotic Life of Property. New York 1983, S. 170f. Ob Hydes Generalisierung dieses Motivs zu einem allgemeinen Strukturprinzip des Gedichtes (,,inhalation and exhala-
} 
Formulierung, die Emerson in Bezug auf Goethe verwendet: „Goethe, a man quite domesticated in the century, breathing its air" ${ }^{43}$ Die übermenschliche Größe des Sprechers (,a kosmos“) ergibt sich also nicht aus einer autochthonen Selbstschöpfung, sondern aus der besonderen Fähigkeit zur universellen Apperzeption.

In dieser Konstellation ist die Vorstellung des Dichters als einer Art Filter der absorbierten Lebenswelt angelegt. ${ }^{44}$ Dieser Gedanke wird hier, in erneutem Anschluss an Emerson, als eine Forderung von allgemeinmenschlicher Gültigkeit zum Ausdruck gebracht: „You shall listen to all sides and filter them from your self“ (V. 28). Das Prädikat „filter“ schließt dabei den Aspekt einer Verwandlung, oder mit Emersons Deutung des Goethe'schen Gedankens gesprochen, einer Erneuerung und Verfeinerung des allumfänglich Wahr- und Aufgenommenen mit ein. Diesen Filterprozess umschreibt Whitman mit der wiederkehrenden Formel „through me“:

Through me many long dumb voices,

Voices of the interminable generations of slaves,

Voices of prostitutes and of deformed persons,

Voices of the diseased and despairing, and of thieves and dwarfs,

Voices of cycles of preparation and accretion,

And of the threads that connect the stars - and of wombs, and of the fatherstuff,

And of the rights of them the others are down upon,

Of the trivial and flat and foolish and despised,

Of fog in the air and beetles rolling balls of dung.

Through me forbidden voices,

Voices of sexes and lusts .... voices veiled, and I remove the veil,

Voices indecent by me clarified and transfigured.

(V. 509-520)

Gerade der Anspruch, die bislang verstummten, die unterdrückten und verbotenen Stimmen der gesellschaftlich Ausgestoßenen in der literarisch-transfigurierenden Rede zum Sprechen zu bringen, deutet auf eine egalitäre, oder, wie Whitman wörtlich sagt, auf eine ,demokratische' Ausrichtung seiner Poetik hin. ${ }^{45}$ Erstaunlicher-

tion $[\ldots]$ are the structuring elements of the poem") wirklich stichhaltig ist, darf allerdings bezweifelt werden.

${ }^{43}$ Emerson: Goethe; or, the Writer, S. 750.

${ }^{44}$,Almost everything in the poem happens as a breathing, an incarnate give-and-take, which filters the world through the body" (Hyde: The Gift, S. 171; meine Hervorhebung).

$45 \mathrm{Vgl}$. hierzu weiterhin grundlegend James Edwin Miller: Leaves of Grass. America's Lyric-Epic of Self and Democracy. New York 1992. Zum Aspekt der Identifikation des Sprechers ,with the lowly and the outcast“ (ebd., S. 41) hält Miller fest: „Whitman's universal embrace appears to be a genuine expression of his profound human - and democratic - sympathies, an emphatic realization in effect of the classic statement by the Latin dramatist Terence [...]: ,I am a man: nothing human is alien to me“" (ebd., S. 42). Die oben angeführte Stelle („Through me forbidden voices“ usw.) liest Miller vor allem 
weise drückt er sich dabei bis in den Wortlaut hinein fast selbst wie Goethe aus, den er in seinen Selbstkommentaren doch ausgerechnet aufgrund seiner vermeintlich selbstbezogenen Grundhaltung verurteilt: „I am of old and young, of the foolish as much as the wise" (V. 326). ${ }^{46}$ An dieser Stelle nur zum Vergleich noch einmal der entsprechende Satz von Goethe: „Zu meinen Werken haben Tausende von Einzelwesen das ihrige beigetragen, Toren und Weise, geistreiche Leute und Dummköpfe, Kinder, Männer und Greise“. Egalitär ist Whitmans poetologische Selbstzuschreibung dabei auch insofern, als er sich dem Kollektiv, das er in sich bündelt, ausdrücklich und immer wieder selbst zurechnet: „I am of“. Dieser explizite Akt der Identifikation findet sich in Goethes Entwurf eines „être collectif“ und seiner Fortschreibung bei Emerson zumindest nicht in dieser Deutlichkeit und ausdrücklich nicht in Bezug auf die bei Whitman genannten Sklaven und Prostituierten, Kranken und Verzweifelten, Diebe und Entstellten.

In anderen Passagen seines Gedichtes treibt Whitman diese Identifikation sogar noch weiter, nämlich hin zu einer vollumfänglichen Transfiguration des Selbst:

I understand the large hearts of heroes,

The courage of present times and all times;

How the skipper saw the crowded and rudderless wreck of the steamship, and death chasing it up and down the storm,

How he knuckled tight and gave not back one inch, and was faithful of days and faithful of nights,

And chalked in large letters on a board, Be of good cheer, We will not desert you;

How he saved the drifting company at last,

How the lank loose-gowned women looked when boated from the side of their prepared graves,

How the silent old-faced infants, and the lifted sick, and the sharplipped unshaved men;

All this I swallow and it tastes good .... I like it well, and it becomes mine,

I am the man .... I suffered ... . I was there.

(V. 817-827)

mit Blick auf die sich aus dem Kotext ergebenden sexuellen Konnotationen, die aber mit dem Aspekt des Politischen gleichsam überblendet werden (vgl. ebd., S. 42-44).

46 An anderer Stelle heißt es, das Ich durchströmten die Stimmen „[o]f the trivial and flat and foolish and despised" (V. 516). 
In diesem zwar nur kleinen, aber strukturell vollumfänglichen Erzähltext ${ }^{47}$ geht es bloß in zweiter Linie um eine nautische Katastrophe. Vor allem wird hier von einer Selbstverwandlung des Sprechers berichtet, die sich über eine körperliche Aufnahme und dadurch vollzogene Aneignung des Geschilderten vollzieht: „All this I swallow and it tastes good .... I like it well, and it becomes mine". Aus dem bloß nachvollziehenden Ich („I understand“) wird so ein leibhaftig erlebendes Ich („I am the man"), und es gibt keinen Grund, diese religiös anmutende Geste der Menschwerdung nicht im buchstäblichen Sinne zu verstehen: Das Ich, das Whitman in seinem Gedicht als Sprecher konzeptualisiert, verwandelt sich in Andere, auch wenn diese Verwandlung nicht immer expliziert, sondern mitunter lediglich konstatiert wird: „I am the hounded slave“ (V. 834), „I am the mashed fireman“ (V. 843). Von hier aus ist es, auch in rezeptionsgeschichtlicher Hinsicht, nicht weit zu dem von Heinrich Detering entschlüsselten Spätwerk von Bob Dylan, dessen transfigurativen Grundzug der Sänger mit Sätzen wie „The people in the songs are all me" auf eine Formel bringt. Den an Whitman erinnernden Charakter dieser Gleichung hebt Detering in der syntaktischen Umkehr des Satzes hervor: „Me are all the people in the songs." 48 In dieser Satzstellung ist die Nähe zwischen Dylans „Me“, Whitmans „I“ und Goethes „Kollektivwesen“ tatsächlich unübersehbar.

Eingeschlossen sind in das Kollektiv der „many long dumb voices“, die den Sprecher durchströmen und die er sich in einem transfigurativen Sinne aneignet, nicht allein die Lebenden, sondern ausdrücklich auch die Toten;49 eine Vorstellung, die in Goethes Rede vom „Kollektivwesen“ nicht explizit vorgesehen ist, ${ }^{50}$ während sie in Emersons Konzept einer Verlebendigung des Vorgefundenen und Aufgenommenen („The record is alive“) zumindest angedeutet wird. Whitmans Poetik läuft demgegenüber auf einen regelrechten Totenkult hinaus, prägnant entfaltet in

\footnotetext{
${ }^{47}$ „Ein Text ist genau dann eine Erzählung“, so lautet die bekannte Minimaldefinition, „wenn er von mindestens zwei Ereignissen handelt, die temporal geordnet sowie in mindestens einer weiteren sinnhaften Weise miteinander verknüpft sind“ (Tilmann Köppe/Tom Kindt: Eræ̌ähltheorie. Eine Einfübrung. Stuttgart 2014, S. 43).

48 Heinrich Detering: Die Stimmen aus der Unterwelt. Bob Dylans Mysterienspiele. München 2016, S. 62 (dort auch das Dylan-Zitat mit Quellenangabe). Anzumerken ist an dieser Stelle, dass in Dylans intellektuellem und künstlerischem Kosmos Whitman ohnehin eine zentrale Stellung zukommt, und dies nicht allein, aber in wesentlicher Hinsicht durch Allen Ginsbergs Vermittlung (hierzu ebd., S. 63).

49 Whitman bezieht dies an anderer Stelle nicht nur auf die ,Stimmen', sondern auf eine organische Transformation im geradezu chemischen Sinne, was in der Forschung bereits mehrfach gesehen worden ist: „,T] he atoms that today compose us once belonged to people of previous generations. Remarkably, these atoms have cycled through multitudes of people stretching back to time and space's inception. The idea that we contain the atoms of multitudes gives new meaning to Whitman's famous line ,I am large .... I contain multitudes“" (Jack Turner: Whitman, Death und Democracy. In: A Political Companion to Walt Whitman. Hg. von John E. Seery. Lexington 2011, S. 272-295, hier S. 275). ${ }^{50} \mathrm{Wohl}$ aber gibt es bei Goethe eine „,Aura“ des eigenhändig Geschriebenen“, wie Schöne betont; er belegt dies mit einem Zitat aus einem Brief an Friedrich Heinrich Jacobi vom 10. April 1812: „Solche Blätter sind mir von unendlichem Werth; denn da mir die sinnliche Anschauung durchaus unentbehrlich ist, so werden mir vorzügliche Menschen durch ihre Handschrift auf eine magische Weise vergegenwärtigt“" (zit. nach Albrecht Schöne: Der Briefeschreiber Goethe. München 2015, S. 11). Vgl. zu derlei Implikationen des Handschriftlichen bei Goethe noch eingehender Sebastian Böhmer: Die Magie der Handschrift. Warum Goethe Autographe sammelte. In: Zeitschrift für Ideengeschichte 5, 4 (2011), S. 97-110.
} 
dem poetologischen Kurzgedicht Pensive and Faltering, das der 1871 veröffentlichten Fassung der Leaves eingegliedert ist:

\author{
Pensive and faltering, \\ The words the Dead I write, \\ For living are the Dead, \\ (Haply the only living, only real, \\ And I the apparition, I the spectre. $)^{51}$
}

Das schreibende Sprecher-Ich gerät hier unversehens zu einer ,Figur des Dritten', die zwischen dem Reich der Lebenden („only living“) und der Sphäre der Hingeschiedenen („the Dead“) vermittelt. ${ }^{52}$ Oder noch etwas genauer: Im Zuge seines animistisch verstandenen, in seiner auratischen Performanz geschilderten Schreibakts (,The words the Dead I write“) regt der Sprecher die Toten zum Widergehen an (,living are the Dead"), und zwar in der Gestalt des Sprechers selbst, der folgerichtig als Geist oder auch Gespenst („I the apparition, I the spectre“) erscheint. Die von Philippe Ariès wirkmächtig beschriebene Tatsache, dass die Religion und mit ihr die Kirche im Zuge der Moderne ihre Alleinstellung als Verwalterin des Totenkults verliert und sich infolgedessen vielfach die Kunst dieser Aufgabe bemächtigt, ${ }^{53}$ betrifft somit auch die Poetik Whitmans.

Aus dem egalitär-demokratischen, auf universelle Inklusion ausgerichteten und zudem totenkultisch aufgeladenen Ansatz Whitmans leitet sich zuletzt ein gesteigerter Anspruch auf Repräsentanz ab, gipfelnd in der berühmtesten und hier bereits zitierten Formel des Gesangs, die einen Zentralbegriff von Emerson aufgreift: „I am large .... I contain multitudes“ (V. 1316). Die semantische Nähe zur Rede vom „Kollektivwesen“ liegt auf der Hand, allerdings identifiziert Whitman das Kollektiv, das bei Goethe mehr oder weniger unspezifisch bleibt, zum einen mit der amerikanische Nation, als deren Sprachrohr er sich versteht: „Walt Whitman, a kosmos, of Manhattan the son" (V. 497). ${ }^{54}$ Zum anderen nennt er die allgemeine Massenhaftigkeit der Moderne als Referenznahmen; eine Massenhaftigkeit, die er durch den Dichter - und damit durch sich selbst - stellvertretend repräsentiert sieht: „And mine a word of the modern .... a word en masse“ (V. 484).

\footnotetext{
51 Whitman: Leaves of Grass, S. 381.

${ }^{52}$ Fassen lässt sich dieser Gedanke unter den von Turner beschriebenen „Inspiration[s] to Creative Immortality“ (Turner: Whitman, Death und Democracy, S. 277-282), die in sämtlichen Spielarten auf einer basalen Auratisierung der Schrift beruhen: „In written words, the self is literally present, whether or not the body that wrote the words is literally living and breathing" (ebd., S. 278).

53 Philippe Ariès: Geschicbte des Todes. München 1980, S. 715-770. Whitman steht in dieser Hinsicht (und vermutlich nur in dieser Hinsicht) in einer Reihe mit Dichtern wie Rainer Maria Rilke, Stéphane Mallarmé, Stefan George oder Ossip Mandelstam, die unter höchst unterschiedlichen literatur- und kulturhistorischen Umständen und in ganz eigener Weise ebenfalls totenkultische Konzeptionen des Gedichts entwickelt haben.

${ }^{54}$ Vgl. hier auch den Schlusssatz im Vorwort zur Erstauflage der Leaves: „The proof of a poet is that his country absorbs him as affectionally as he has absorbed it." (Whitman: Leaves of Grass, S. 636)
} 
In der Übersicht zeigt sich, dass sich die Nähe des im Song of Myself entworfenen Ich zu Emersons und Goethes Ansätzen in gleich mehreren Aspekten niederschlägt: in der Nicht-Originalität des Dichters und seiner Abhängigkeit von der sinnlich wahrgenommenen und in sich aufgenommenen Lebenswelt; in der Umwandlung der absorbierten Lebenswelt in Kunst; in der universellen und zugleich egalitären Ausrichtung sowie dem impliziten Anspruch des Dichters auf Repräsentanz. Dass hierbei zumindest partiell von einer indirekten Einflussbeziehung auszugehen ist, zeigt sich nicht nur in der überschneidenden Lexik (Emerson: „,breathing its air", Whitman: „breathe the air"). Mehr noch und auf konzeptueller Ebene lässt sich dies an Whitmans romantisierender Funktionalisierung des Goethe'schen Modells erkennen. Emersons Lesart des „Kollektivwesens“, vor allem aber der Auftrag, den er aus diesem Konzept herleitet und im Schlusssatz seines Goethe-Essays zusammenfasst, liest sich diesbezüglich fast wie eine argumentative Vorlage:

We too must write Bibles, to unite again the heavenly and the earthly world. The secret of genius is $[\ldots]$ to realize all that we know; in the high refinement of modern life, in arts, in sciences, in books, in men, to exact good faith, reality, and a purpose; and first, last, midst, and without end, to honor every truth by use. 55

Der literarische Text als eine heilige Schrift, die syntheseartig verbinden soll, was in der modernen Welt in einer unübersichtlichen, unübersehbaren Vielheit zerspalten vorliegt, so bestimmt Emerson den kompensatorischen Auftrag des modernen Dichters. Whitman folgt ihm dabei im Grundsatz nach, wenn er die Dichter als „gangs of kosmos" und „prophets en masse“ bestimmt. ${ }^{56}$ Sie erscheinen bei ihm als Vermittler des Verschiedenen (,arbiter of the diverse" ${ }^{67},{ }^{57}$ als Erfüller des zu Erfüllenden (,,supplies what wants supplying $\left.{ }^{6}\right),{ }^{58}$ dies nun allerdings in nationalpatriotischer Zuspitzung, was bereits im Vorwort zur Erstauflage der Leaves zum Ausdruck gebracht wird:

Of all Nations the United States with veins full of poetical stuff most need poets and will doubtless have the greatest and use them the greatest. Their Presidents shall not be their common referee so much as their poet shall. [...] He is the arbiter of the diverse and he is the key. He is the equalizer of his age and land $\ldots$ he supplies what wants supplying $[\ldots] .{ }^{59}$

\footnotetext{
${ }^{55}$ Emerson: Goethe, or the Writer, S. 761.

${ }^{56}$ Whitman: Leaves of Grass, S. 634.

${ }^{57}$ Ebd., S. 620.

${ }^{58}$ Ebd.

${ }^{59}$ Ebd., S. 619-620.
} 
Zu diesem Anspruch passte es zunächst, dass Whitman die 1860 erschienene dritte Edition der Leaves in ihrer materialen Gestalt einer einfach gebundenen King James-Bibel des späten 19. Jahrhunderts annäherte. ${ }^{60}$ Allerdings darf hierbei nicht das charakteristische Spannungsverhältnis der Konstellation aus dem Blick geraten: Der Dichter als Vermittler des Unterschiedenen - das bedeutet eben keine Aufhebung des Mannigfachen zugunsten einer neuen und allumfassenden Totalität. Whitman scheint es vielmehr um einen intermediären Ansatz zu gehen, wie er ähnlich bei Goethe und Emerson konzeptualisiert wird (und nicht voreilig über den Begriff der Organizität entproblematisiert werden sollte), ${ }^{61}$ nämlich um die Vermittlung von Einzelnem und Ganzem, Heterogenität und Harmonie, Vielheit und Einheit.

Vor dem Hintergrund der hier vollzogenen Rekonstruktion stellt sich die eingangs aufgeworfene Frage, warum Whitman einen Einfluss Goethes nicht nur verschwieg, sondern ausdrücklich von sich wies, mit gesteigerter Dringlichkeit. Die Antwort hierauf wird man nicht allein in Whitmans inhaltlicher Kritik an Goethe finden, sondern, mehr noch, in Goethes Stellung als paradigmatischem Vertreter des europäischen Geisteslebens. Denn so eindringlich Whitman die Forderung nach einer autochthonen amerikanischen Literatur formuliert - mit ihm selbst als ihrem ersten und zugleich hervorragendsten Vertreter -, so unvermeidlich geht damit die Zurückweisung ihrer abendländischen Wurzel einher: „Still further, as long as the States continue to absorb and be dominated by the poetry of the Old World [...], so long will they stop short of first-class Nationality and remain defective." 62 Die bemerkenswerte, auffällige Vehemenz, mit der sich Whitman von Goethe absetzt, erweist sich vor diesem Hintergrund als eine nationalpatriotische Spielart der von Harald Bloom beschriebenen Anxiety of Influence. ${ }^{63}$ Tatsächlich aber gilt für diesen Fall: Brooklyn und Weimar trennen keine Welten - es bedurfte nur eines Umwegs über Concord, Massachusetts.

\footnotetext{
${ }^{60}$ Walt Whitman: Leaves of Grass, 1860. The 150th Anniversary Facsimile Edition. Hg. von Jason Stacey. Iowa City 2009, vgl. hierzu im Vorwort des Herausgebers S. x.

${ }^{61}$ Ich beziehe mich hier auf ein naheliegendes Missverständnis. Der Begriff der ,organischen Demokratie', der in der Whitman-Forschung vielfach kursiert, bezieht sich auf ein bestimmtes Set an Werten und Normen, die als Teil des menschlichen Wesens und als Grundlage des sozialen Miteinanders erachtet werde. Von einer ,organischen Kultur' geht Whitman jedoch keineswegs aus, wie auch Mack betont: „While it is true that Whitman was moved by an appreciation for the interconectedness of life, it would nevertheless be a profound error to confuse the concept of organic democracy with the notion of an organic American state: they are radically different, even antithetical, ideas" (Stephen John Mack: The Pragmatic Whitman. Reimagening American Democracy. Iowa City 2002, S. 160-165, hier S. 163).

62 Whitman: Leaves of Grass, S. 584 (A Backward Glance O'er Travel'd Roads).

${ }^{63}$ Harald Bloom: The Anxiety of Influence: A Theory of Poetry. New York 1973.
} 